\title{
HF Wire-Mesh Dipole Antennas for Broadband Ice-Penetrating Radar
}

\author{
Jonathan D. Hawkins, Student Member, IEEE, Lai Bun Lok, Paul V. Brennan and Keith W. Nicholls
}

\begin{abstract}
A novel high frequency (HF) to very high frequency (VHF) wire-mesh dipole antenna design for use in polar regions is discussed and evaluated. The antenna was designed to be lightweight, readily demountable, and acceptably robust. This is an initial step in the development of a ground-based, phasesensitive, synthetic aperture imaging system for use on an autonomous rover platform. The results of initial trials on the Rhône Glacier, Switzerland in August 2019 are discussed, with particular attention being paid to the effect on antenna performance of high surface water content. Including the effects of surface water resulted in good agreement between field results and modelled performance.
\end{abstract}

Index Terms-dipole antennas, HF/VHF radar, ice-penetrating radar

\section{INTRODUCTION}

Radar systems used to investigate and monitor glaciers in polar and alpine regions have operated predominantly over the same HF and VHF frequency bands since initial experiments in the 1960s [1]. It is well established that the propagation of radio waves through homogeneous, cold ice is effectively frequency independent over these frequency bands [2]. There has been a trend for ice-penetrating radar systems to move towards airborne surveys which are able to produce data with higher spatial coverage than their ground-based counterparts. Airborne HF platforms have successfully sounded temperate ice [3], [4], however the physically large wavelengths relative to the size of the airborne platform mean that antennas cannot be mounted on the airborne platform directly and are often deployed in the form of towed monopoles. To reduce the effect of off-nadir clutter, VHF and UHF ultra wideband (UWB) radars have been developed which make use of antenna arrays to improve directivity [5]. These radars have improved vertical resolution compared to their HF counterparts at the cost of increased Friis losses and transmitter signal powers.

The evolution of ice shelf features such as meltwater channels [6], basal terraces [7] or basal crevasses is not well understood but plays an important role in ice-ocean interactions and ice-shelf stability. Combining a phase-sensitive radar such as the ApRES (autonomous phase-sensitive radio echo sounder) [8] with an autonomous rover platform has the potential to enable large scale synthetic aperture radar (SAR) imaging of ice shelves in order to study temporal changes

This work was supported by the Royal Society Research Fellows Enhancment Award RGS\EA \180173

J. D. Hawkins and P. V. Brennan are with the Radar Group, Department of Electronic \& Electrical Engineering, University Collge London, London, WC1E 7JE, UK (e-mail: jonathan.hawkins.17@ucl.ac.uk).

L. B. Lok is with the Department of Engineering, Lancaster University, Lancaster, LA1 4YW, UK.

K. W. Nicholls is with the British Antarctic Survey, Cambridge, CB3 0ET, in basal topography. While ground penetrating radar (GPR) systems operating in the UHF bands and above are suited to integration with autonomous rovers because of the small size of antennas required for these radars, such systems are better suited to near surface imaging as a result of increased Friis and dielectric losses at microwave frequencies [2]. Because the maximum spatial sampling interval required in order to apply SAR processing techniques is proportional to the radar wavelength [9], the scale of ground-based surveys possible with VHF or UHF radars is limited to ensure that both the spatial Nyquist sampling criterion is met and the survey is completed in a reasonable time.

The purpose of this paper is to discuss the development of a broadband antenna suitable for use with a modified HF/VHF version of the ApRES deployed on an autonomous rover. The operating bandwidth of the HF/VHF prototype is reduced from $200-400 \mathrm{MHz}$ to $20-40 \mathrm{MHz}$ which increases the upper-bound of the maximum along-track sample spacing allowed for SAR processing techniques to be used without introducing aliasing [10]. This enables the measurement of temporal changes in basal topography of ice shelves at ten times the rate of an equivalent platform using the VHF/UHF ApRES. However, since the antenna size tends to increase as the radar frequency is reduced, integration of an HF/VHF radar system with an autonomous rover presents an engineering challenge - particularly if the system is to be used in areas with complex surface topography. Because existing autonomous rovers have been developed for use across various scientific missions, GPR have often been deployed as towed payloads [11], [12] rather than directly integrated on to the rover.

The remainder of this paper is structured as follows. Section II reviews the theoretical limits on operating bandwidth and antenna size to provide a benchmark and initial design goal for the HF/VHF ApRES antenna, followed by a review of existing broadband HF/VHF antennas. Section III introduces a wire-mesh dipole design that takes into consideration constraints imposed by use in glaciated environments. Section IV discusses the characteristics of the wire-mesh dipole antenna measured during a field campaign in August 2019 and the changes made to the antenna model to account for deployment on a temperate glacier.

\section{BROADBAND HF/VHF ANTENNAS}

The bandwidth requirements of a radar antenna are determined by the desired down-range resolution $\Delta R$ of the radar system, given by the following

$$
\Delta R=\frac{\lambda_{0}}{2 B_{f} \sqrt{\varepsilon_{r}}}
$$


where $B_{f}$ is the fractional bandwidth $B / f_{0}, B$ is the absolute bandwidth, $f_{0}$ is the centre frequency, $\lambda_{0}$ is the wavelength at the centre frequency in free space and $\varepsilon_{r}$ is the relative permittivity of the medium through which the signal propagates. For the HF/VHF ApRES, $\Delta R=4.19 \mathrm{~m}$. In [13], Chu establishes a limit on the fractional bandwidth for an arbitrary antenna determined by its largest physical dimension. The limit was later updated and modified by McLean [14] to include a parameter for the antenna radiation efficiency $\mu$ as follows

$$
\frac{1}{B_{f}} \approx Q=\mu\left(\frac{1}{(k r)^{3}}+\frac{1}{k r}\right)
$$

where $Q$ is the quality factor of the antenna, $k$ is the wavenumber $2 \pi / \lambda_{0}$ and $r$ is the radius of the sphere that minimally bounds an antenna. It can be seen from (2) as the antenna dimension becomes smaller, the quality factor increases and hence the bandwidth reduces. The definition of quality factor is frequency dependent and hence the relationship between $Q$ and $B_{f}$ fail when considering UWB (low- $Q$ ) antennas. [15] shows that, when the fractional bandwidth used corresponds to the geometric half-power fractional bandwidth, (2) is still valid. The geometric fractional bandwidth for the HF/VHF ApRES is $B_{f}^{\prime}=\sqrt{2^{-1}}$. Following [15], the longest wavelength $\lambda_{\max }$ from the operating bandwidth is used in place of $\lambda_{0}$. Solving (2) to find $r$, the minimum antenna dimension for a lossless antenna $(\mu=1)$ with a $20 \mathrm{MHz}$ to $40 \mathrm{MHz}$ bandwidth in free space is $5.72 \mathrm{~m}$. Other considerations for the design of antennas to be deployed in polar environments are that they should be compact, lightweight and robust to facilitate transport and continuous operation. The use of wiremesh as a reflector has been common since the development of some of the earliest radar systems [16], [17], however more recently there has been interest in using wire-mesh structures as the radiating element to design lightweight [18] or optically transparent antennas [19]. The flexibility and reduced weight of wire-mesh makes it suitable for the construction of an HF/VHF antenna for ice-penetrating radar.

From [15] the design goal is to maximise the intrinsic $3 \mathrm{~dB}$ bandwidth of the radiating element, which is to be improved using a passive matching network. An example at $\mathrm{HF}$ is the twin-terminated folded dipole antennas developed for the SuperDARN space weather radar network which make use of an optimised $N^{\text {th }}$ order $L C$ matching network [20]. An alternative broadband antenna design commonly used in GPR is the resistively-loaded dipole antenna [21], [22]. While effective for pulsed radar systems because of the high peak powers involved, the use of resistors inherently results in reduced antenna efficiency.

\section{Design And Simulation}

The proposed design is a planar 'fat dipole' to be constructed from two rectangular sheets of centre-fed wire-mesh (Fig. 1). Before considering the operating conditions and matching requirements of the antenna, a comparison was made in simulation between solid 'fat' dipole elements and their wire-mesh equivalent in free space.

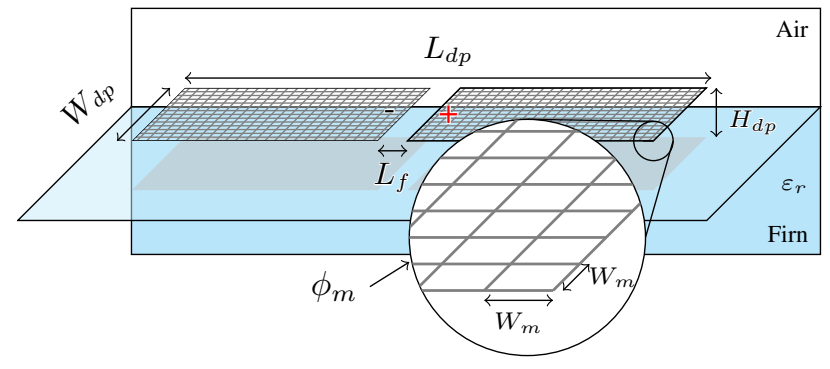

Fig. 1: Schematic of the wire-mesh dipole antenna for simulation in dielectric half-space model of a glacier.

TABLE I: Antenna model parameters and values.

\begin{tabular}{|c|c|c|l|}
\hline Parameter & VHF/UHF $(\mathrm{m})$ & HF/VHF $(\mathrm{m})$ & Description \\
\hline$L_{d p}$ & 0.5 & 5 & Dipole length \\
$W_{d p}$ & 0.09 & 0.9 & Dipole width \\
$H_{d p}$ & 0 & 0.1 & Height above dielectric interface \\
$L_{f}$ & 0.03 & 0.3 & Feed length \\
$W_{m}$ & 0.006 & 0.006 & Mesh cell size \\
$\phi_{m}$ & 0.0006 & 0.0006 & Mesh diameter \\
\hline \hline$\varepsilon_{r}$ & 1 & {$[1.6, \ldots, 3.2]$} & "Firn" permittivity (unitless) \\
\hline
\end{tabular}

\section{A. Verification of Wire-Mesh Approach}

The wire-mesh fat dipole antenna in Fig. 1 was modelled and simulated using SIMULIA CST Microwave Studio ${ }^{\circledR}$. For each of the commercially available mesh cell sizes $\left(W_{m}=6\right.$, $13,25$ and $50 \mathrm{~mm})$ with fixed wire diameter $\left(\phi_{m}=0.6 \mathrm{~mm}\right)$, the reflection coefficient $(\Gamma)$ of the wire-mesh antenna was compared with the simulated $\Gamma$ from an equivalent planar fatdipole using solid radiating elements. For all $W_{m}$ less than or equal to $25 \mathrm{~mm}$, the RMS error between the wire-mesh and solid radiator reflection coefficients was less than $10 \%$. For these simulations the dipole height $H_{d p}$ was set to zero and $\varepsilon_{r}=1$ such that the simulation space is symmetrical and homogeneous. Parameter values used in the simulation are given in Table I. Subsequent simulations of the wiremesh dipole were performed using the solid radiator equivalent model to reduce the simulation complexity and run time. The initial lengths of the dipole $\left(L_{d p}\right)$ were chosen to be $0.5 \mathrm{~m}$ for the VHF/UHF scale-model and $5 \mathrm{~m}$ for the HF/VHF antenna corresponding to one half-wavelength in free-space. It follows from (2) that the antennas will fail to meet the fractional bandwidth criteria of $\sqrt{2^{-1}}$ for use with the ApRES without the introduction of a matching network.

Simulations were conducted to test the effect of tapering the angle at the feedpoints of the antenna, similar to a bowtie design. While an increased taper (up to $70^{\circ}$ ) resulted in a reduction of the resonant frequency of the antenna by up to $2.1 \mathrm{MHz}$, it increased the reactive impedance and hence reduced the $3 \mathrm{~dB}$ bandwidth. Therefore it was decided to continue with the rectangular, untapered design.

\section{B. Operating Environment}

The presence of metallic and dielectric objects in the nearfield of an antenna has the potential to alter its operating behaviour. In a glacial environment, snow is blown or falls on the surface of a glacier and densifies over time to become glacial ice. "Firn" refers to the material in the intermediate 


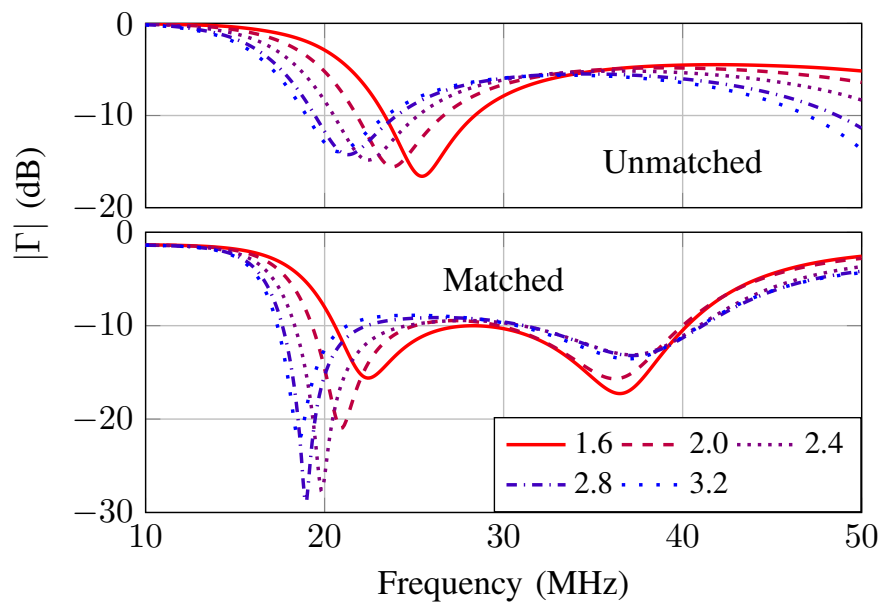

Fig. 2: Variation in $|\Gamma|$ for an unmatched and matched wiremesh dipole antenna positioned in dielectric half-space of increasing permittivity $\left(\varepsilon_{r}\right)$.

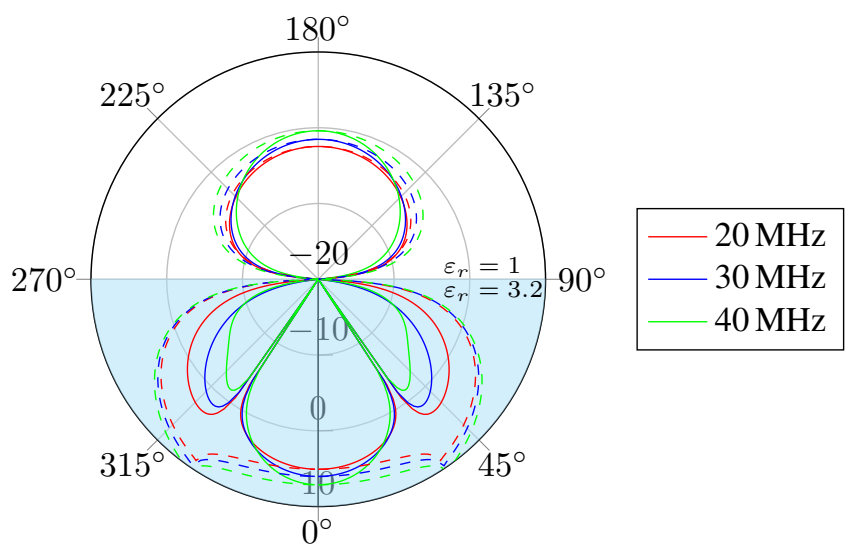

Fig. 3: Far-field directivity (dBi) in $E$-plane (solid) and $H$ plane (dashed) for the wire-mesh dipole antenna.

stage between fresh snow and glacial ice. The density $\rho$ of firn varies from $350 \mathrm{~kg} \mathrm{~m}^{-3}$ for wind-packed snow to $923 \mathrm{~kg} \mathrm{~m}^{-3}$ for glacial ice [23], corresponding respectively to relative permittivities of 1.67 and 3.16 calculated using an empirical relationship [24].

To assess the changes in antenna characteristics (such as reflection coefficient and far-field radiation pattern), the permittivity of the dielectric half-space described in Fig. 1 was varied between 1.6 and 3.2 in intervals of 0.4 . Variation in the simulated reflection coefficient is shown in Fig. 2. Increasing the permittivity of the dielectric half-space, equivalent to the antenna being situated on denser firn, reduces the resonant frequency of the antenna and increases the transmitted power.

The far-field radiation pattern was calculated using the nearto-far-field transformation and is shown for $20 \mathrm{MHz}, 30 \mathrm{MHz}$ and $40 \mathrm{MHz}$ in Fig. 3. The radiation pattern agrees with that of a dipole positioned on a dielectric half-space [22]. The mean directivity across the bandwidth at nadir increases from $4.0 \mathrm{dBi}$ to $6.1 \mathrm{dBi}$ as $\varepsilon_{r}$ is increased from 1.6 to 3.2. For all simulated values of $\varepsilon_{r}$, the antenna directivity is dominated by the $H$ plane with a peak directivity varying from $8.6 \mathrm{dBi}$ to $9.7 \mathrm{dBi}$ at $\pm 53^{\circ}$ when $\varepsilon_{r}=1.6$, decreasing to $\pm 34^{\circ}$ as $\varepsilon_{r}$ is increased to

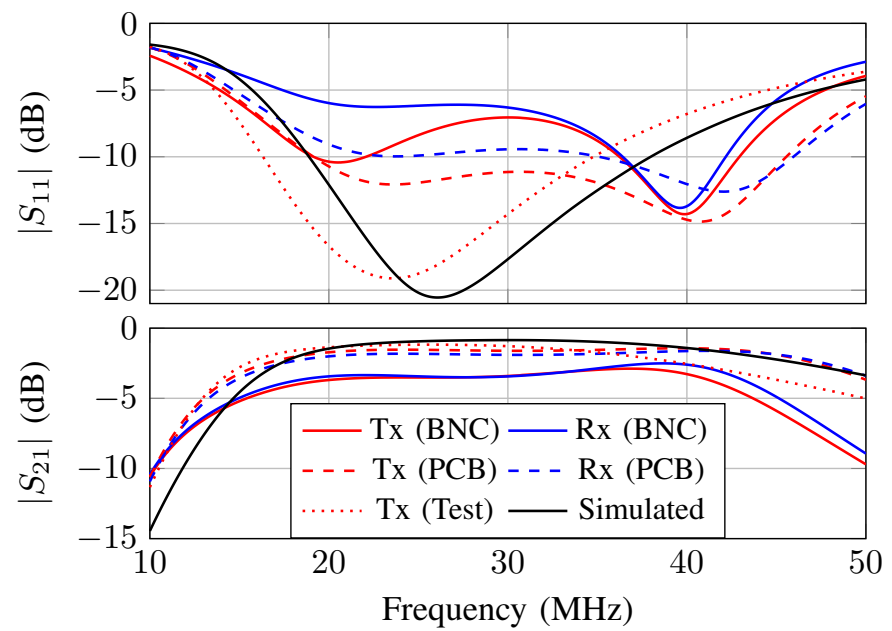

Fig. 4: Comparison of measured and simulated $\left|S_{11}\right|$ and $\left|S_{21}\right|$ for the wire-mesh dipole matching network.

3.2. Hence, clutter in the $H$-plane is more likely to dominate the radar range profile than clutter in the $E$-plane.

\section{Matching Network}

A $5^{\text {th }}$-order $L C$ matching network was implemented on the antenna side of a $50 \Omega$ 1:1 balun in order to maximise the power transmission to the wire-mesh dipole antennas. The matching network was designed and optimised using the simulated antenna impedance for various permittivities (or firn densities) in the Keysight ADS Design Environment ${ }^{\circledR}$. A sensitivity analysis of the matched $\Gamma$ identified the two most sensitive components which were realised with variable capacitors to allow for the matching network to be tuned when the antenna is positioned on various types of firn or glacial ice. Fig. 2 compares the unmatched and matched reflection coefficient of the wire-mesh dipole antenna when positioned in a dielectric half-space with variable permittivity. In each case the matching network was optimised for the appropriate value of permittivity and satisfies $\left|S_{11}\right|$ less than $-8 \mathrm{~dB}$ and $\left|S_{21}\right|$ greater than $-2 \mathrm{~dB}$ over the $20 \mathrm{MHz}$ to $40 \mathrm{MHz}$ bandwidth.

\section{MEASUREMENTS}

The HF/VHF wire-mesh dipole antennas were tested on the Rhône Glacier, Switzerland (46³5'12'N, 8²3'16”E) during a field campaign to trial the capabilities of the HF/VHF ApRES in August, 2019. Each of the wire-mesh dipoles were supported on a demountable $40 \mathrm{~mm}$ diameter PVC pipe rectangular structure which allowed the antenna to be transported and rigidly deployed in the field (Fig. 5). Each assembled antenna had a gross weight less than $4 \mathrm{~kg}$. The $s$-parameters for the matching networks and antennas were measured using a calibrated vector network analyzer (DG8SAQ). Fig. 4 compares the simulated and measured $s$-parameters for the matching network at various connector reference planes in the balun housing shown in Fig. 5 (inset). These were at the external BNC connectors, PCB SMA connectors and an onboard test SMA connector. The reflection coefficients for each antenna, shown in Fig. 6, measured on the Rhône Glacier with 


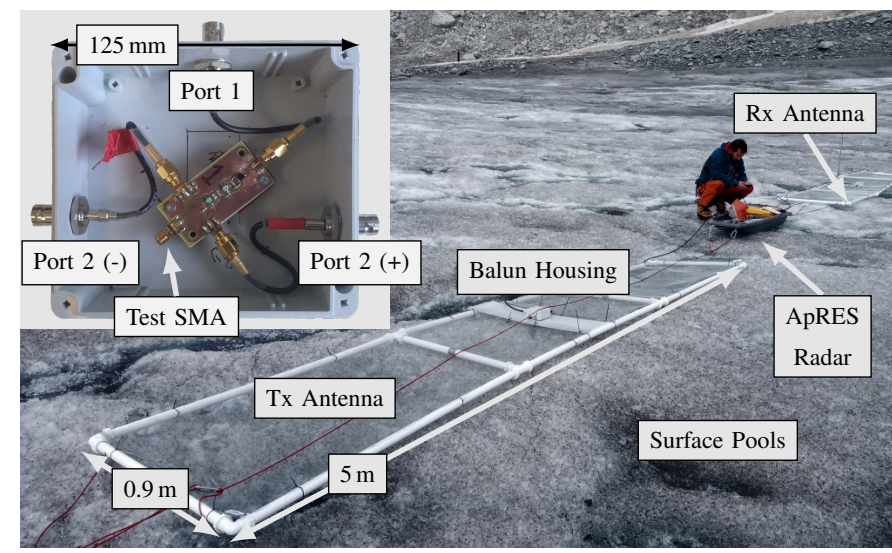

Fig. 5: System setup with sled-mounted radar and wire-mesh dipole antennas. Inset: balun housing with matching network PCB, on-board test SMA and external BNC connectors.

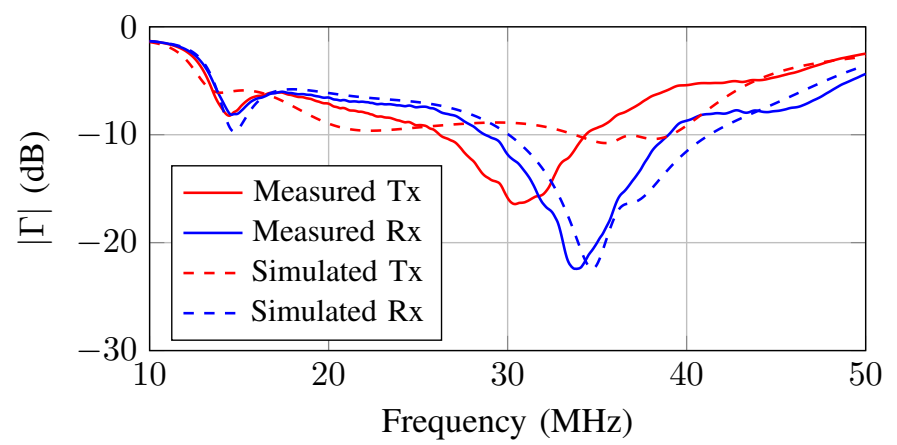

Fig. 6: $|\Gamma|$ of both wire-mesh dipole antennas measured on the Rhône Glacier, compared with modelled behaviour of a wiremesh dipole antenna situated on waterlogged alpine glacier.

over $30 \mathrm{~m}$ separation, disagree with the modelled results in Fig. 2. Some variation between the results for the transmit and receive antennas is attributed to non-uniformity in the manufacturing of the matching networks as seen in Fig. 4, however the resonances that are now apparent at $30 \mathrm{MHz}$ and $34 \mathrm{MHz}$ require further analysis. Simulations of both antennas separated at their phase centres by $9.3 \mathrm{~m}$ as during radar operation (Fig. 5) showed no significant effect on the reflection coefficient.

\section{A. Analysis}

Before field deployment of the antennas, there was $40 \mathrm{~mm}$ rainfall on the glacier over the preceding two days. The field site for testing the antennas was relatively flat and waterlogged. The presence of surface pools (visible in Fig. 5), indicates that englacial water is likely to be stored and transported along grain boundaries [25] but restricted to the upper tens of centimetres in the glacier.

Approximating the waterlogged ice as a fractional proportion $v$ of spherical inclusions of water in glacial ice, the Looyenga equation (3) can be used to model its permittivity [26]. The values used for water and glacial ice are assumed to be $\varepsilon_{w}=80$ and $\varepsilon_{i}=3.18$ respectively. The loss tangent of freshwater is low therefore the dielectric can be treated as lossless. The simulation represented in Fig. 1 is modified to introduce a secondary dielectric layer between the air-firn interface with thickness $H_{w}$, representing the waterlogged glacier surface, and permittivity $\varepsilon$.

$$
\varepsilon^{\frac{1}{3}}-\varepsilon_{i}^{\frac{1}{3}}=v\left(\varepsilon_{w}^{\frac{1}{3}}-\varepsilon_{i}^{\frac{1}{3}}\right)
$$

A comparison of the measured antenna results is generated by combining the measured balun $s$-parameters with the simulated reflection coefficient of the saturated glacier antenna model as shown in Fig. 6. A minimum mean squared error (MMSE) process with $H_{w}$ and $v$ as independent variables was used to estimate the model parameters for the transmit and receive antennas, with results shown in Table II. Swapping the matching network $s$-parameters for the transmit and receive antennas did not yield significant changes in MMSE, however the antennas were not in identical positions for both measurements on the glacier which suggests some disagreement in Fig. 6 can also be attributed to a change in surface water distribution. If multiple antenna calibration measurements were to be taken during a radar traverse, it would be possible to approximate the spatial variation in surface water content with this method. Increased surface melt has recently been reported across Antarctica [27] and Greenland [28], therefore accounting for waterlogged and temperate conditions in the matching network design is likely to be necessary for future polar deployments of the HF/VHF ApRES.

\section{B. Future Work}

Further refinement to the passive matching network is required to improve the matched antenna bandwidth. A reduction in the transmission line lengths from the matching network PCB to external connectors is likely to result in more consistent matching performance between antennas. A more robust antenna frame beyond the existing PVC pipe structure should also be considered for integrating the HF/VHF ApRES with an autonomous rover for long endurance RES surveys.

\section{CONCLUSION}

A novel HF/VHF antenna design for use in ice-penetrating radar surveys has been presented. The use of wire-mesh as radiating elements was shown to match simulated results and allows for the construction of a lightweight antenna suitable for rapid field deployment. The design was verified through simulation and experimentally on an alpine glacier in wet summer conditions, and was used to conduct a successful radar transect of the glacier with the HF/VHF ApRES. The matching network design and a modified glacier model with a waterlogged surface can be used to explain the reduction in measured bandwidth. With increased surface melt seen in both polar regions, consideration of the effects of supraglacial water is likely to be an important factor when considering integration of the radar platform and an autonomous rover.

TABLE II: Parameters for MMSE optimisation of $|\Gamma|$ between measured and modelled wire-mesh dipole on saturated glacier.

\begin{tabular}{|c|c|c|l|}
\hline Antenna & $H_{w}(\mathrm{~m})$ & $v(\%)$ & MMSE \\
\hline Tx & 0.4 & 50 & 0.073 \\
Rx & 0.1 & 30 & 0.038 \\
\hline
\end{tabular}




\section{ACKNOWLEDGMENT}

JDH and LBL thank their colleagues from ETH-Zurich (Mauro Werder and Greg Church) and Cardiff University (Liz Bagshaw and Mike Prior-Jones) for facilitating the fieldwork on Rhône Glacier.

\section{REFERENCES}

[1] S. Evans, "Radio techniques for the measurement of ice thickness," Polar Record, vol. 11, no. 73, pp. 406-410, 1963.

[2] S. Fujita, T. Matsuoka, T. Ishida, K. Matsuoka, and S. Mae, "A summary of the complex dielectric permittivity of ice in the megahertz range and its applications for radar sounding of polar ice sheets," Physics of Ice Core Records, pp. 185-212, 2000. [Online]. Available: https: //eprints.lib.hokudai.ac.jp/dspace/bitstream/2115/32469/1/P185-212.pdf

[3] E. Rignot, J. Mouginot, C. F. Larsen, Y. Gim, and D. Kirchner, "Lowfrequency radar sounding of temperate ice masses in Southern Alaska," Geophysical Research Letters, vol. 40, no. 20, pp. 5399-5405, 2013.

[4] E. Arnold et al., "HF/VHF radar sounding of ice from manned and unmanned airborne platforms," Geosciences (Switzerland), vol. 8, no. 5, pp. 1-22, 2018.

[5] F. Rodriguez-Morales et al., "Advanced multifrequency radar instrumentation for polar Research," IEEE Transactions on Geoscience and Remote Sensing, vol. 52, no. 5, pp. 2824-2842, 2014.

[6] A. M. Le Brocq et al., "Evidence from ice shelves for channelized meltwater flow beneath the Antarctic Ice Sheet," Nature Geoscience, vol. 6, no. 11, pp. 945-948, 2013.

[7] P. Dutrieux, C. Stewart, A. Jenkins, K. W. Nicholls, H. F. J. Corr, E. Rignot, and K. Steffen, "Basal terraces on melting ice shelves," Geophysical Research Letters, vol. 41, no. 15, pp. 5506-5513, aug 2014. [Online]. Available: http://doi.wiley.com/10.1002/2014GL060618

[8] K. W. Nicholls, H. F. J. Corr, C. L. Stewart, L. B. Lok, P. V. Brennan, and D. G. Vaughan, "Instruments and methods: A ground-based radar for measuring vertical strain rates and time-varying basal melt rates in ice sheets and shelves," Journal of Glaciology, vol. 61, no. 230, pp. 1079-1087, jul 2015. [Online]. Available: https://www.cambridge.org/ core/product/identifier/S0022143000200269/type/journal \{\_\}article

[9] N. Holschuh, K. Christianson, and S. Anandakrishnan, "Power loss in dipping internal reflectors, imaged using ice-penetrating radar," Annals of Glaciology, vol. 55, no. 67, pp. 49-56, 2014.

[10] I. G. Cumming and F. H. Wong, "Digital processing of synthetic aperture radar data: algorithms and implementation," 2005.

[11] G. Koh, J. Lever, S. A. Arcone, H. Marshall, and L. Ray, "Autonomous FMCW radar survey of Antarctic shear zone," Proceedings of the 13th Internarional Conference on Ground Penetrating Radar, GPR 2010, 2010.

[12] J. Lever, A. Delaney, L. Ray, E. Trautmann, B. L.A., and A. Burzynski, "Autonomous GPR Surveys using the Polar Rover Yeti," Journal of Field Robotics, vol. 30, no. 2, pp. 194-215, 2013.

[13] L. J. Chu, "Physical limitations of omni-directional antennas," Journal of Applied Physics, vol. 19, no. 12, pp. 1163-1175, 1948

[14] J. S. McLean, "A re-examination of the fundamental limits on the radiation Q of electrically small antennas," IEEE Transactions on Antennas and Propagation, vol. 44, no. 5, pp. 672-676, may 1996. [Online]. Available: http://ieeexplore.ieee.org/document/496253/

[15] H. Schantz, The Art and Science of Ultra-Wideband Antennas, 2nd ed. Artech House, jun 2015.

[16] E. J. Sterba, "Directive antenna system," nov 1932.

[17] H. Griffiths and N. Willis, "Klein Heidelberg-The First Modern Bistatic Radar System," IEEE Transactions on Aerospace and Electronic Systems, vol. 46, no. 4, pp. 1571-1588, 2010.

[18] T. Warnagiris, "The "Chicken Wire Wonder" - A Unique Broadband Vertical Antenna for the HF Bands," Tech. Rep., 2009.

[19] S. H. Kang and C. W. Jung, "Transparent Patch Antenna Using Metal Mesh," IEEE Transactions on Antennas and Propagation, vol. 66, no. 4, pp. 2095-2100, 2018

[20] E. Custovic, H. Q. Nguyen, J. C. Devlin, J. Whittington, D. Elton, A. Console, H. Ye, R. A. Greenwald, D. A. Andre, and M. J. Parsons, "Evolution of the SuperDARN antenna: Twin Terminated Folded Dipole antenna for HF systems," IB2COM 2011 - 6th International Conference on Broadband Communications and Biomedical Applications, Program, pp. 24-29, 2011.

[21] T. Wu and R. King, "The Cylindrical Antenna with Nonreflecting Resistive Loading," IEEE Transactions on Antennas and Propagation, vol. 13 , no. 6 , p. $998,1965$.
[22] S. A. Arcone, "Numerical studies of the radiation patterns of resistively loaded dipoles," Journal of Applied Geophysics, vol. 33, no. 1-3, pp. 39-52, 1995.

[23] K. M. Cuffey and W. S. B. Patterson, The physics of glaciers., 4th ed., W. S. B. Paterson, Ed. Burlington, Mass.: Burlington, Mass., 2010.

[24] A. Kovacs, A. J. Gow, and R. M. Morey, "The in-situ dielectric constant of polar firn revisited," Cold Regions Science and Technology, vol. 23, no. 3, pp. 245-256, may 1995. [Online]. Available: https://www.sciencedirect.com/science/article/pii/0165232X9400016Q

[25] A. G. Fountain and J. S. Walder, "Water flow through temperate glaciers," Reviews of Geophysics, vol. 36, no. 3, pp. 299-328, 1998.

[26] J. W. Glen and J. G. Paren, "The Electrical Properties of Snow and Ice," Journal of Glaciology, vol. 15, no. 73, pp. 15-38, 1975.

[27] J. Kingslake, J. C. Ely, I. Das, and R. E. Bell, "Widespread movement of meltwater onto and across Antarctic ice shelves," Nature, vol. 544, no. 7650 , pp. $349-352,2017$.

[28] A. K. Kendrick et al., "Surface Meltwater Impounded by Seasonal Englacial Storage in West Greenland," Geophysical Research Letters, vol. 45, no. 19, pp. 10,410-474,481, oct 2018. [Online]. Available: https://doi.org/10.1029/2018GL079787 